\title{
Research on the Architecture and Governance Mechanisms of a Civil-military Integration Technology Innovation Network*
}

\author{
Tiejun Jiang \\ Department of Management Engineering and Equipment Economics \\ Naval University of Engineering \\ Wuhan, China
}

\author{
Chengjie Zhou \\ Department of Management Engineering and Equipment \\ Economics \\ Naval University of Engineering \\ Wuhan, China
}

\author{
Huaiqiang Zhang \\ Department of Management Engineering and Equipment \\ Economics \\ Naval University of Engineering \\ Wuhan, China
}

\begin{abstract}
The development of a civil-military integration technology innovation network is an effective way to promote civil-military integration technology innovation and boost the deep-integrated development of national defense science and technology industry and civil industry. In the process of civilmilitary integration technology innovation, it is particularly critical to fully identify the architecture of an innovation network and effectively promote its formation and efficient operation. On the basis of the network theory model of Swedish Network School, the architecture of a civil-military integration technology innovation network is systematically analyzed. Considering its unique characteristics and practical problems and following the logical route of its formation and operation, the governance model including governance objectives, governance environment, network state and governance mechanisms is constructed. Based on the principle of conciseness, the governance mechanisms such as trust, coordination, profit and competition are designed. Considering the development needs of the network, the measures for realization of various mechanisms are further proposed.
\end{abstract}

Keywords-civil-military integration; governance mechanism; network architecture; technology innovation network

\section{INTRODUCTION}

With the globalization of economy and science and technology, around the global flow and reorganization of science and technology resources, various types of technology innovation networks have gradually been formed. The current researches generally think that a technology innovation network is a network organization that includes universities, research institutes, companies, governments,

*This work was supported in part by the Military Science Project of National Social Science Foundation of China under Grant NO.16GJ003 105 and the Autonomous Project of Naval University of Engineering under Grant NO.20161632 financial institutions and public institutions. These nodes which are designed for technology innovation form interactive relationships through contractual or informal arrangements and participate in technology development and diffusion [1] [2].

In the context of civil-military integration, in order to solve outstanding issues such as uncertainty of technology innovation, scarcity of resources and limited ability of innovative organizations under the modern innovative environment, help innovation organizations make better use of external resources, strengthen core capabilities and achieve innovation objectives, additionally, to benefit all network members by improving the competitiveness of a network and the ability to resist risks overall, military and civil companies, universities, research institutes, government, troops and various intermediary organizations have formed a civil-military integration technology innovation network [3]

A civil-military integration technology innovation network can effectively exert the advantages of organizations, realize the optimal allocation of resources, reduce the innovation cost, shorten the innovation time, reduce the innovation risk, expand the access to information and resources, increase the strategic flexibility and greatly promote the rapid development of civil-military technology. However, in the process of development, there are also some problems that make it difficult to form and operate a technology innovation network, lead to the crisis between network organizations and ultimately cause adverse effects on technology innovation activities. In terms of network formation, there exist problems such as sector separation, closed operation, mechanism differences, opinion divergence, system lags, trade monopolization, standard thresholds and financial constraints [4]. In terms of network operation, there exist problems such as self-protection, opportunism and path dependence [5]. 
Therefore, in the process of civil-military integration technology innovation, it is particularly critical to fully identify the architecture of a technology innovation network and effectively promote its formation and efficient operation. Effective governance mechanisms are ways to solve these problems and promote the role of a technology innovation network [6] [7] [8].

The researches on governance mechanisms of network organizations are extensive. For example, on the basis of expanding the transaction cost economy theory, Jones constructed a four-dimensional trading environment for network governance and proposed a theoretical model of network governance based on social mechanisms [7]. Jones's governance model was used as a framework to analyze the governance issue of a military and civil technology transfer network, and the governance mechanisms such as trust, learning, ecology and culture were proposed [8] [9]. In view of the problems such as conservatism, free-riding and unilateral dependence in an enterprise technology innovation network, the governance mechanisms such as trust, comortgage, power and incentive were proposed [10] [11] [12]. Based on a survey of data from 100 companies in the sensor technology industry, three different types of governance mechanisms in open innovation networks and their effects on joint innovation generation were analyzed [13] [14]. From the current research situation, the specific mechanisms involved in the governance of network organizations include trust, learning, reputation, distribution, innovation, decisionmaking, coordination, sanctions, culture, incentives and constraints [15] [16] [17].

At present, the problems existing in the researches on governance mechanisms in the field of civil-military integration technology innovation are mainly reflected in: 1) Most researches are limited to enterprise technology innovation networks, which is difficult to reflect the unique formation and operation of a civil-military integration technology innovation network. 2) The analysis based on Jones's governance model mainly considers governance objectives, governance environment and governance mechanisms, but does not include changes in network state caused by governance mechanisms. However, these changes will have important impacts on the application of governance mechanisms. 3) Most researches focus on the analysis on mechanisms themselves and lack the consideration of the measures for their realization. 4) The proposed governance mechanisms are relatively more, but in fact many mechanisms are intersected, that is, some mechanisms can be mutually realized.

In this paper, on the basis of analyzing the architecture of a civil-military integration technology innovation network, considering its characteristics and problems and following the logical route of its formation and operation, a governance model including governance objectives, governance environment, network state and governance mechanisms is established and effective governance mechanisms and the measures for their realization are proposed based on the principle of conciseness.

\section{NETWORK ARCHITECTURE}

\section{A. Network Composition}

According to the network theory model proposed by Håkansson, a well-known scholar of Swedish Network School, a technology innovation network includes three basic elements: actors (or participants, nodes), resources and activities (or connections) [20] [21]. These elements together constitute the architecture of a civil-military integration technology innovation network, as shown in "Fig. 1". 


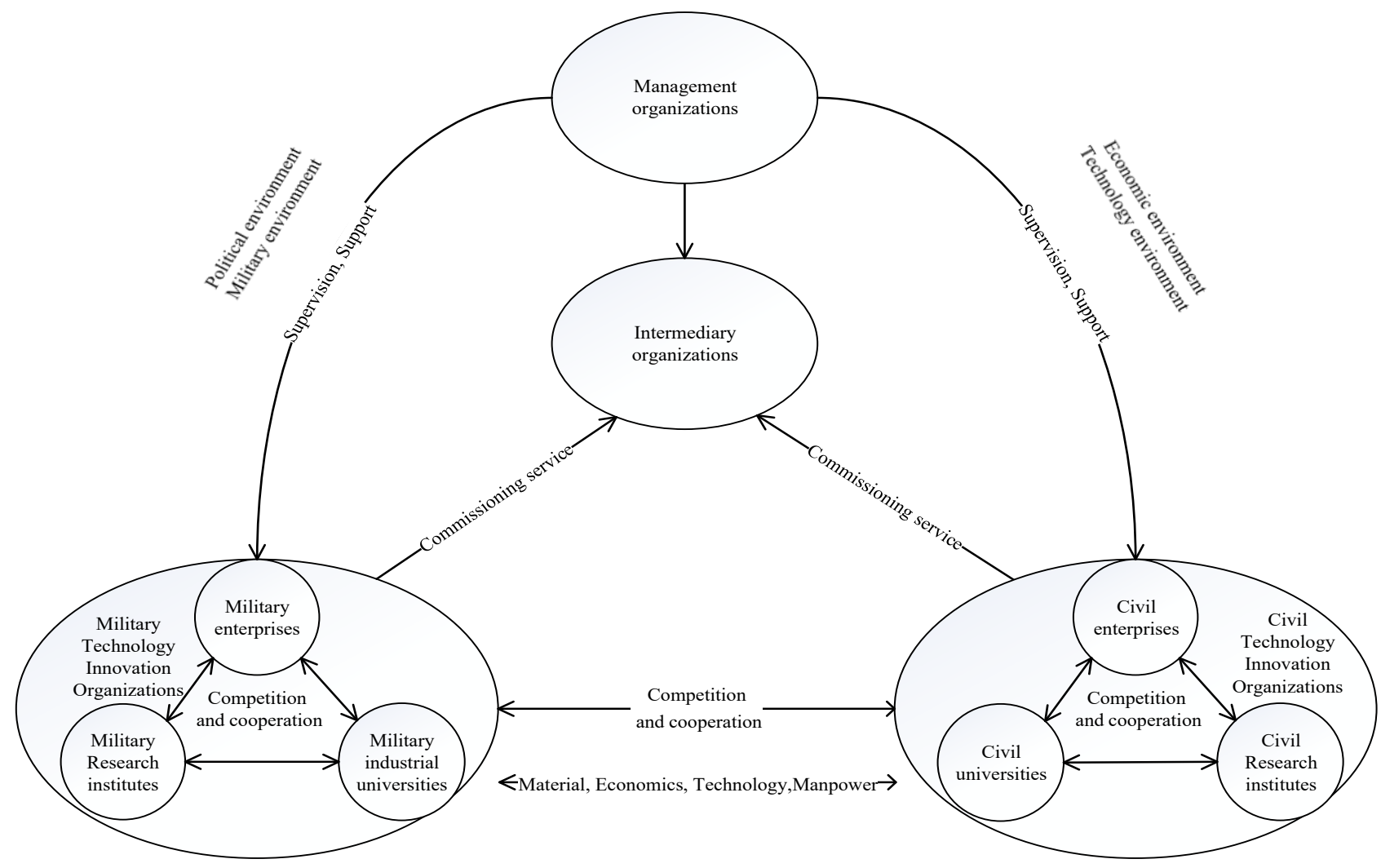

Fig. 1. The architecture of a civil-military integration technology innovation network.

\section{B. Network Actors}

The network actors are various types of organizations that are closely related to technology innovation activities, have clear role orientation, cooperate with each other based on the division of labor, perform their own functions consciously or in accordance with established rules and form harmonious interaction relationships.

According to the organizational affiliation and industry division, the actors in a civil-military integration technology innovation network can be divided into the following four categories:

1) Military technology innovation organizations: They are the main body and core of an innovation network and the main source of military technologies, including military industry groups and subordinate enterprises, military research institutes and some civil defense science and technology universities. Their achievements are mainly used for national defense construction.

2) Civil technology innovation organizations: Like military technology innovation organizations, they are also the main body and core of an innovation network and the main sources of civil technologies, including various types of private enterprises, civil universities and research institutes.

3) Management organizations: As the leader and maintainer of civil-military integration technology innovation activities, they include local government departments and military-related management departments. Their main function is to maintain and regulate technology innovation environment and coordinate the relationship among actors in a technology innovation network.

4) Intermediary organizations: They are the adhesives among technology innovation organizations, which serve as the supply medium for various resources and the bridge between suppliers and demanders of cooperative technology innovation. According to their property right, they can be divided into government intermediary organizations, intermediary organizations within national defense science and technology industry, other non-profit social organizations and enterprise organizations specialized in technical innovation consulting services, etc.

\section{Network Resources}

The network resources, as the foundation for the formation and operation of a civil-military integration technology innovation network, mainly include:

1) Material resources: They are the key facilities and equipment owned by civil-military technology innovation organizations, innovative platforms with professional influence including national, provincial and ministerial-level scientific research bases, key laboratories and key engineering centers.

2) Economic resources: They are the technology innovation funds owned by civil-military technology innovation organizations including the research and 
development funds provided by the relevant national departments according to the plan and the funds obtained by some technology innovation organizations through financing channels.

3) Technology resources: They are the knowledge and technology owned by civil-military technology innovation organizations, such as intellectual property rights (patents, copyrights, etc.).

4) Human resources: They are professional technical and management personnel owned by civil-military technology innovation organizations.

\section{Network Connections}

In a civil-military integration technology innovation network, various actors interact with each other to form network connections. In summary, there are mainly four types: cooperation, competition, management and service.

1) Cooperation: Cooperation is the main connection between organizations in an innovation network, and it is also an important source of network formation. In an innovation network, each innovation organization interacts through a series of formal and informal cooperation relationships and carries out innovation cooperation with complementary advantages and joint investment. It not only realizes resource sharing, but also spreads risks, improves innovation capability and efficiency, so that cooperation can achieve a win-win situation. There are two main types of cooperation: technology innovation resource transfer, technology innovation task allocation or resource sharing. Because an innovation network contains a large number of related organizations, the above-mentioned situations will coexist in the network, and the innovative cooperation behavior between them will inevitably be complex and diverse.

2) Competition: The formation of a technology innovation network promotes the optimal allocation of innovative resources. In addition, the introduction of competition is also one of the core objectives. Competition is an important source of vitality for a technology innovation network. The competition within a technology innovation network runs through the entire process of innovation activities, involving the initial investment of resources, intermediate development of technologies, finally access to markets and service for troops. Forming and maintaining an orderly competitive atmosphere is a necessary condition for maintaining the vitality of a technology innovation network and promoting its efficient and healthy development.
3) Management: Management is the basic activity to ensure the timely formation and orderly operation of a technology innovation network. Its main implementer is the management organization. Management includes designing and implementing management rules of a technology innovation network, providing innovation conditions and deploying innovation resources. In the construction of a technology innovation network, management organizations usually only participate in technology innovation activities indirectly, mainly through the implementation of management, promote the formation of a technology innovation network, enhance the civil-military integration of technology innovation, and create conditions for maximizing network benefits.

4) Service: Services are auxiliary activities in the formation and operation of a technology innovation network. They are usually completed by intermediary organizations, and some of them are also implemented by management organizations. Services include information exchange, technology diffusion and transfer, transformation of scientific and technological achievements, assessment and appraisal of science and technology, allocation of innovation resources, management consulting, etc. These activities have led to close internal relations among various types of innovation organizations and management organizations. Services do not directly participate in technology innovation, but they play a unique role in promoting the rapid transformation of scientific and technological achievements, reducing innovation cost, improving the transaction efficiency of innovation results and effectively avoiding innovation risks.

\section{GOVERNANCE MODEL}

The governance model of a civil-military integration technology innovation network mainly includes governance objectives, governance environment, network state and governance mechanisms, as shown in "Fig. 2".

\section{A. Governance Objectives}

According to the basic logical route of the formation and operation of a technology innovation network, the governance objectives are established. At the same time, in terms of the operation of the network, two levels of governance objectives, namely the basic and normal operation, efficient and orderly operation, have been proposed.

The governance objectives are embodied as follows: 


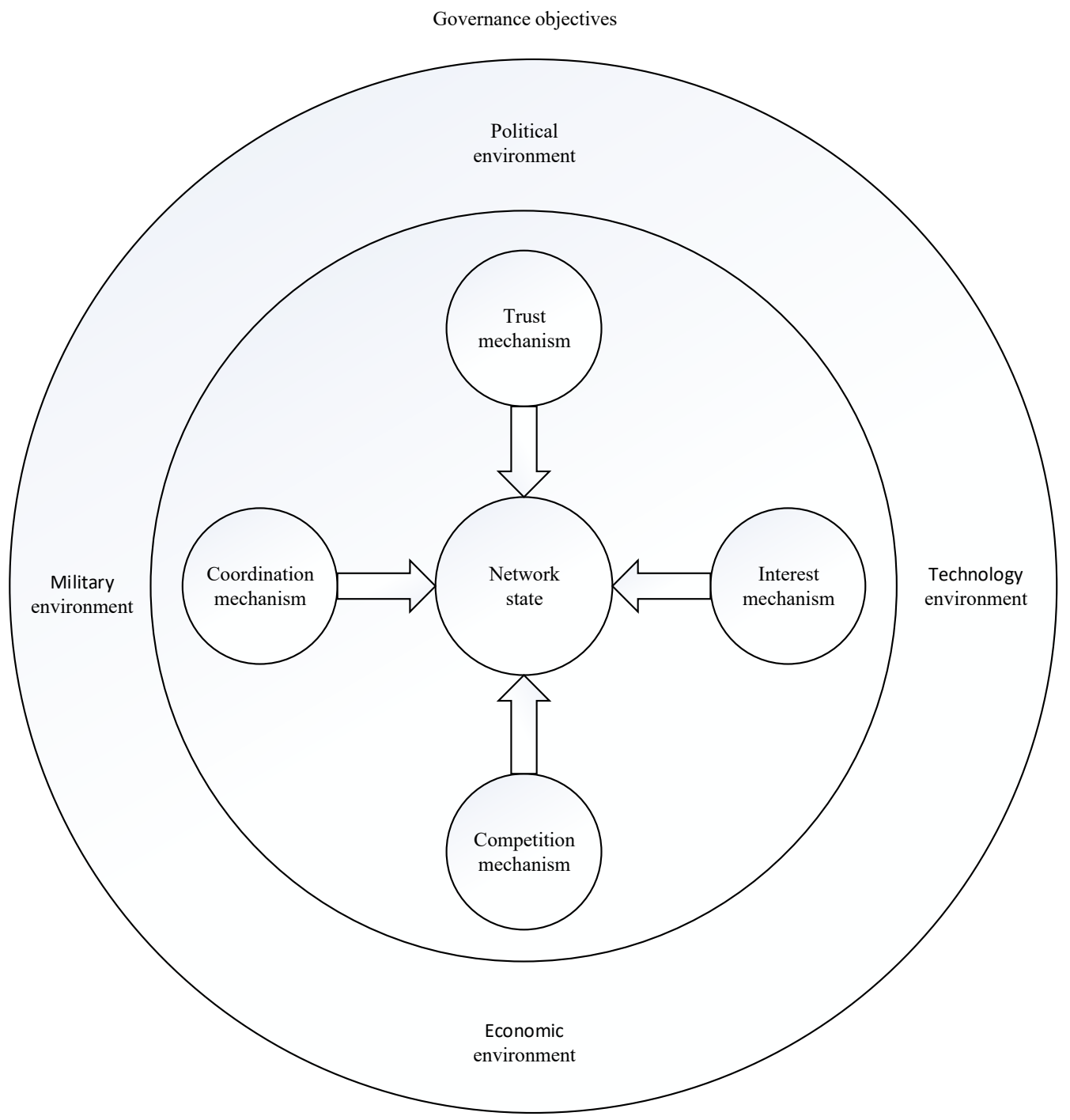

Fig. 2. The composition of the governance model of a civil-military integration technology innovation network.

1) Promoting the rapid formation of the network: The advantages of a technology innovation network require the extensive participation of different types of organizations, especially military and civil technology innovation organizations. Fully activating the potential of the innovation network and mobilizing active participation of all kinds of organizations is the primary governance objective of the network.

2) Maintaining the normal operation of the network: After all kinds of organizations enter the network, good operation environment and perfect operation rules are needed to ensure the normal operation of the network.

3) Improving the efficiency and efficiency of the network: On the basis of the normal operation of the network, it is especially necessary to improve the efficiency and efficiency of network operation by optimizing mechanism design.

\section{B. Governance Environment}

A civil-military integration technology innovation network is in a complex and changeable environment. The network should give full play to the effectiveness of governance mechanisms and adjust timely governance mechanisms to adapt to changes in the environment. The governance environment mainly includes:

1) Political and military environment: All these require the timely adjustment of an innovation network, giving full play to the integration advantages and adapting to the development and change of military needs. This kind of environment is the unique governance environment that a civil-military integration technology innovation network needs to face.

2) Science and technology environment: They are embodied in the accelerated technical progress, the increasingly obvious technical systematization features, the increasing complexity of a technical system and the 
enhanced technical comprehensiveness. All of these require the integration of military and civil technology innovation networks to meet the needs of technology innovation.

3) Economic environment. They are embodied in the process of economic globalization, rapid market changes and increasingly fierce market competition. The contradiction between the fund demand and supply of military science and technology innovation is becoming increasingly prominent. All these require an innovation network to actively respond to changes in the economic environment.

\section{Network State}

It refers to the realization state of a technology innovation network to the governance objectives through the function of governance mechanisms under the established governance environment, which reflects the adaptability of a technology innovation network to governance environment, the effect of governance mechanisms and the realization degree of governance objectives.

For network state, it can be seen from two aspects: in terms of the positive effect, it is manifested as the guarantee of technology innovation activities and the realization degree of governance objectives; in view of the negative effect, it shows the main problems, types and degree of problems in the formation and operation of the network and the distance from the governance objectives.

The core objective of network governance is to solve some problems existing in the formation and operation of a technology innovation network, however, these problems will vary from time to time because of changes in the external environment and the role of governance mechanisms. Therefore, the application of all kinds of governance mechanisms should be adjusted according to the specific network state in order to strengthen the pertinence of network governance and make the network always in a state of gradual improvement.

\section{Governance Mechanisms}

Regarding the proposed governance model, governance mechanisms specifically refer to a set of guidelines for guiding technology innovation behaviors of various organizations, which is established in response to some issues in the formation and operation of a technology innovation network, based on preset governance objectives and a certain governance environment. They can be generalized into four mechanisms, namely trust, coordination, profit and competition.

1) Trust mechanism: It refers to technology innovation network organizations regulate their own technology innovation behaviors in a highly self-conscious manner within the established institutional framework. Trust is the fundamental governance mechanism of a technology innovation network. To maintain the formation and operation with trust is the ideal state of a network. The trust mechanism includes basic mechanisms such as reputation. Specifically, technology innovation network organizations are willing to enter the network to participate in technology innovation activities. Moreover, in the process of joint technology innovation, the organizations have full confidence in each other and a good cooperation atmosphere is formed.

2) Coordination mechanism: One of the core issues of a technology innovation network is the coordination between organizations. The prominent difference between a technology innovation network and a single organization innovation is the large increase of various organizations, resulting in a significant increase in the difficulty of coordination. The coordination mechanism will play an important role in promoting the realization of organizational objectives and the resolution of conflicts. The coordination mechanism includes common constraints, sanction and other mechanisms.

3) Profit mechanism: Profit is one of the core objectives of various organizations to participate in a technology innovation network. The profit mechanism plays an important role in promoting the rapid formation of the network and maintaining its good operation. The profit mechanism mainly includes two aspects: firstly, innovation organizations are guided into the network by profit and the network is formed; secondly, the profit of innovative organizations is ensured, the network is functioning properly and its efficacy can be achieved. The profit guidance includes the mechanisms of learning, cooperation and incentive, etc., and the profit guarantee includes the allocation mechanism.

4) Competition mechanism: Competition is the product of market economy. Therefore, the competition mechanism plays an important role in the operation of the network. Competition is an important prerequisite for maintaining the healthy operation of an innovation network and improving its efficiency and efficiency.

\section{MEASURES FOR REALIZATION OF GOVERNANCE MECHANISMS}

The established governance model and the proposed governance mechanisms provide a theoretical guarantee for network governance. However, if governance mechanisms are reflected in an actual innovation network, it is also necessary to select scientific approaches and design effective measures to realize them by aiming at the characteristics of mechanisms.

\section{A. Trust Mechanism}

The measures for realization of the trust mechanism mainly include:

1) Understanding and recognition of institutional mechanisms and culture: The premise of trust is understanding. The various organizations in a civil-military integration technology innovation network come from civilmilitary technology innovation systems. The differences between institutional mechanisms and culture of different 
systems are relatively large. Differences in understanding are likely to occur between two organizations, resulting in a sense of mistrust and further affecting the development of technology innovation activities. Specific solutions to these problems include: strengthening the propaganda and exchange of civil-military technology innovation environment, promoting both sides to understand each other's innovative institutional mechanisms and culture, and removing obstacles to understanding.

2) Creating opportunities for the communication between network organizations: There is a certain correlation between the strength of trust relationships and the frequency of interactions. In order to establish and deepen trust relationships between network organizations, it is necessary to create as many communication opportunities as possible between network organizations and to deepen mutual understanding through frequent contacts. Specific measures include: breaking the situation in which civilmilitary technology innovation organizations obstruct information and act independently, establishing and improving a two-way information exchange platform to realize the transmission of information and resources between innovation organizations and expanding application fields of military and civil technologies.

3) Establishing open and fair system norms: The establishment of a trust relationship pursues without resort to system constraints, but the establishment of open and fair system norms has an important role in promoting the establishment of conscious behavior and the formation of a trust relationship. Specific measures include: establishing fair and trustworthy codes of conduct and norms jointly understood and respected by a military and civil technology innovation system and adopting a strategy of self-initiated and external assurance of innovative organizations to create the conditions for the establishment and maintenance of trust relationships as much as possible.

\section{B. Coordination Mechanism}

The measures for realization of the coordination mechanism mainly include:

1) Establishing perfect operation rules: There are many problems that need to be solved during the operation of a technology innovation network, for example, the identification of rights and responsibilities of various organizations, the specification of cooperation relationships and the definition of intellectual property. These require the establishment of relevant specific rules and the introduction of practical and feasible solutions to address possible moral hazard, adverse selection and other issues in the network. The establishment of perfect operation rules can greatly reduce the coordination, reflect the fair and just and decrease the difficulty of coordination, which is helpful to the efficient development of cooperative innovation and provide a system guarantee for the effective realization of the coordination mechanism. Specific measures include: clarifying the respective responsibilities and obligations of network organizations, formulating sound working procedures and methods for dealing with related issues.

2) Dealing with contradictions and conflicts: The incomplete design and the non-standard realization process of network operation mechanisms may lead to the occasional failure of the operation rules. It is necessary to control and resolve contradictions and conflicts that occur in the process of network operation. For the coordination problem that cannot be completely solved by current operation rules, it is usually necessary to introduce thirdparty governance, that is to say, a separate entity department is introduced to specifically coordinate network organizations and their mutual relations, to objectively analyze the causes of contradictions and conflicts to form a solution and to govern coordination problems in the network to ensure the realization of network objectives.

\section{Profit Mechanism}

The measures for realization of the profit mechanism mainly include:

1) Profit guidance: The profit guidance mainly refers to that the profit drive is used to attract various organizations to participate in technology innovation activities and promote the formation of the network through the optimization design of system. Specific measures include:

- In terms of policy, military and civil technology innovation organizations are given the same status, and even in some technology innovation activities, civil technology innovation organizations are given a more prominent position, such as by adjusting military special project research plans to provide the support for dual-use items.

- In terms of technology, civil technology innovation organizations are provided with science and technology resources such as military test facilities, so that they can enjoy technical support and reduce their special investment.

- In terms of capital, civil technology innovation organization is given certain financial and tax advantages, and financial subsidies are given in key development areas. Defense research funds have been established to provide the financial support for the participation of civil technology innovation organizations in the basic research of national defense technology. Financial instruments have been flexibly applied to broaden the financing channels of civil technology innovation organizations.

2) Profit assurance: The profit guarantee means to ensure the profit of network organizations which are obtained by participating in technology innovation, so as to stimulate the enthusiasm and creativity of network organizations to participate in technology innovation and maintain the healthy operation of a technology innovation network. Specific measures include: 
- In terms of profit distribution, a fair and equitable profit distribution system should be established. The profit distribution plan of the full performance of a contract and the profit distribution method of the incomplete performance of a contract should be made clear through a standard contract to guarantee the profit of partners.

- In terms of property management, through the establishment of a sound and property system of science and technology, the profit relationship between various organizations should be adjusted from the legal level, technology innovation achievements under the condition of market economy should be guaranteed, which can promote the mutual exchange and common progress in the military and civil field.

- In terms of innovation supervision, the perfect supervision mechanism of technology innovation should be established as soon as possible, in order to strengthen the innovation process supervision, give full play to the supervisory role, such as the prevention, detection and identification of possible illegal, discipline, irregularities in the process of technology innovation, protect the profit of participants, reduce their participation risks and ensure the benefit of cooperative technology innovation.

\section{Competition Mechanism}

The measures for realization of the competition mechanism mainly include:

1) Introducing competition: The introduction of competition is to introduce a certain number of innovative organizations that are homogenized into a technology innovation network and moderately create a competitive atmosphere through the system design. Specific measures include:

- In terms of policy, in the research field where technology monopoly is strong, exclusive operation is serious, and it is of great significance to military and civil applications, new research institutions should be gradually nurtured and supported; At the same time, non-competition determination of cooperative objects should be restrained in cooperation technology innovation activities, and non-competition declaration and approval system should be established.

- In terms of technology, the barriers to participation in technology innovation should be cleared, military standards and norms should be restricted, and civil standards should be used as much as possible, so as to promote the generalization of technology innovation foundation, reduce the cost of cooperative technology innovation, make civil technology innovation organizations into military technology innovation and pave the way for the promotion of competition system.
2) Maintaining competition: The maintenance of competition is to ensure a certain number of alternative technical innovation organizations to keep the competitive situation by establishing or maintaining alternative sources of technology innovation, to take some balancing measures in the competition to protect the survival and development of technology innovation organizations and to ensure the benefits of both sides in the competition. Specific measures include:

- In terms of competition losers, through laws, contractual stipulations or administrative constraints, competition winners should be applied to certain intervention that losers are allowed to participate in some technical cooperation in order to reduce the loss of losers and maintain their technology, talent reserves in the related field. In general, the competitive environment is maintained.

- In terms of organizational merger behaviors, they should be encouraged when they are conducive to the healthy operation of a technology innovation network, otherwise, they should be imposed appropriate intervention, through the relevant policies and regulations to prevent the formation of an industry monopoly and to ensure that at least two units of technology innovation in some areas can participate in the competition.

- In terms of unfair competition, the system of appeal adjudication should be established and unfair competition behaviors should be defined in the relevant regulations. Technical innovation organizations should be allowed to complain about the disputes in the competition and the channels, procedures and arbitration departments of the complaint should be made clear to maintain the fair and impartial competition.

\section{CONCLUSION}

It is an effective way to develop a civil-military integration technology innovation network, which is used to strengthen the integration of military and civil technology and promote the in-depth integration of national defense science and technology industry and civil industry. There are many problems in the formation and operation of an innovation network, which has its inevitability. To design some effective governance mechanisms and ensure their effective realization will promote the continuous development of a technology innovation network and give full play to the potential advantages of an innovation network.

Based on the principle of complementary, compatibility and coordination, innovation organizations in the military and civil system will integrate advantage resources under the function of governance mechanisms. With the establishment of an innovation network and its healthy, orderly and efficient development, the maximum value of a civil-military integration technology innovation will be realized. 


\section{REFERENCES}

[1] R. W. Rycroft. Technology-based globalization indicators: the centrality of innovation network data $[\mathrm{J}]$. Technology in Society, 2003, 25(3): 299-317.

[2] G. Keith Cambron BS MS. Integration and innovation [M]. Global Networks: Engineering, Operations and Design. John Wiley \& Sons, Ltd, 2012: 249-263.

[3] X. Li, X. Gu, X. C. Guo. Study on evolution of civil-military integration industrial cluster based on innovation network theory $[\mathrm{C}] / /$ Mechanics and Mechanical Engineering: Proceedings of the 2015 International Conference, 2015: 652-659.

[4] J. Liang. Research about technological innovation with deep civilmilitary integration [C]// International Conference on Social Science and Technology Education, 2015: 979-983.

[5] I. Anthony. The evolution of dual-use technology controls: a historical perspective [M]. Technology Transfers and NonProliferation: Between Control and Cooperation, 2014.

[6] K. Janzen, B. Hillebrand, G. W. Ziggers, et al. An explorative study on what network governance in innovation networks entails: towards a typology [J]. Human Resource Development Review, 2011.

[7] K. G. Provan, A. Fish, J. Syd. Literature on whole networks interorganizational networks at the network level [J]. Journal of Management, 2007, 33(3): 479-516.

[8] C. Assens, A. C. Lemeur. Network governance: the theory [M]. Networks Governance, Partnership Management and Coalitions Federation. Palgrave Macmillan UK, 2016.

[9] C. Jones, S. W. Hesterly, P. S. Borgatti. A general theory of network governance: exchange condition and social mechanisms [J]. Academy of Management Review, 1997, 22(4): 911-945.

[10] X. W. He, G. M. Hou, Y. Wang. Analysis of civil-military technology transfer network governance [J]. Scientific Management Research, 2012, 30(2): 91-95. (In Chinese)

[11] F. Xin, M. Wei. Study on the governance mechanism of technology enterprise based on the improvement of independent innovation ability [J]. Management Science \& Engineering, 2010, 4(4): 106-112.

[12] J. Gao, Z. C. Mi, J. B. Wei. Research on governance mechanism of enterprise technology innovation network [J]. Science \& Technology Progress and Policy, 2007, 24(9): 133-136. (In Chinese)

[13] Y. Xie, H. Zhang. The empirical research on relationship between the relational governance of members based on resources dependence and core enterprise formation in technology innovation networks $[\mathrm{C}] / /$ International Conference on Business Management and Electronic Information. IEEE, 2011: 403-406.

[14] H. Scarbrough, K. Amaeshi. Knowledge governance for open innovation: evidence from an EU R\&D collaboration [J]. Knowledge Governance, 2009: 220-246.

[15] T. Clauß, P. Spieth. Open innovation networks: governance mechanisms for enhancing innovation generation [C]// Strategic Management Society International Conference, 2015.

[16] Clauß T, Spieth P. Governance of open innovation networks with national vs. international scope $[\mathrm{J}]$. Journal of Strategy \& Management, 2017, 10(1): 66-85.

[17] W. A. Li. Network organization - new trends in organization development [M]. Economic Science Press, 2001. (In Chinese)

[18] L. Ying. A mechanism framework for cluster enterprise innovation: a view of co-governance [C]// International Conference on Management of E-Commerce and E-Government. IEEE, 2008: 283288.

[19] M. Bevir, R. A. W. Rhodes. Decentred theory, change and network governance [M]. Theories of Democratic Network Governance. Palgrave Macmillan UK, 2007.

[20] H. Håkansson, I. Snehota. No business is an island: The network concept of business strategy $[\mathrm{J}]$. Scandinavian Journal of Management, 2006, 22(3): 256-270.

[21] H. Håkansson, A. Waluszewski. A never ending story: interaction patterns and economic development [J]. Industrial Marketing Management, 2013, 42(3): 443-454. 